\title{
Hydrogen passivation of boron acceptors in as-grown boron-doped CVD diamond epilayers
}

\author{
C. Fernández-Lorenzo a,*, D. Araújo ${ }^{\text {b }}$, J. Martín ${ }^{\text {a }}$, R. Alcántara a ${ }^{\text {, J. Navas }}{ }^{\text {a }}$, M.P. Villar ${ }^{\mathrm{b}}$, M.P. Alegre ${ }^{\mathrm{b}}$, \\ P.N. Volpe ${ }^{\mathrm{c}}$, F. Omnès ${ }^{\mathrm{c}}$, E. Bustarret ${ }^{\mathrm{c}}$ \\ a Dpto. Química Física, Facultad de Ciencias, Universidad de Cádiz, 11510 Puerto Real, Cádiz, Spain \\ b Dpto. Ciencia de los Materiales e IM y QI, Facultad de Ciencias, Universidad de Cádiz, 11510 Puerto Real, Cádiz, Spain \\ ' Institut Néel, CNRS-Université Joseph Fourier, 25 av. des Martyrs, 38042-Grenoble, France
}

\section{A R T I C L E I N F O}

Available online 25 February 2010

\section{Keywords:}

Diamond

Boron doping

Cathodoluminiscence

FTIR

\begin{abstract}
A B S T R A C T
A homoepitaxial boron-doped diamond single layer is investigated by means of Fourier transformed infrared spectroscopy (FTIR) and cathodoluminescence (CL). Both techniques are shown to be complementary. $\mu$-FTIR mapping allows to determine the location of active boron while CL allows discernability between passivation and compensation. Hydrogen incorporation during chemical vapour deposition (CVD) growth is revealed to passivate boron acceptors. The obtained results highlight that plasma etching can induce a dissociation of $\mathrm{B}-\mathrm{H}$ centres.
\end{abstract}

(c) 2010 Elsevier B.V. All rights reserved.

\section{Introduction}

Since the attribution of p-type doping to boron acceptors, strong variations in the diamond properties depending on the growth conditions (blue colour for gemological application, carrier densities, defect densities, superconducting critical temperature Tc, etc.) have been repeatedly reported in the scientific and technical literature over the last twenty years. However, the way in which boron is incorporated within the diamond lattice is still an open question. Indeed, the formation of hydrogen-acceptor complexes with point defects, boron pairs and clusters or interstitial sites can lead to electrically inactive boron incorporation and to an impurity-induced damage to the crystalline quality which may, for instance, explain the brown colour of metallic CVD epilayers. Such kind of defects should reduce the mobility of the carriers without improving their density. The way in which the hydrogen passivates the boron acceptor is also an open question. Recently, Barjon et al. [1] reported the difference in the reactivation of boron after deuterium and hydrogen related passivation. They found that $\mathrm{B}-\mathrm{H}$ complexes are twice more stable than B-D ones. This behaviour seems to be the inverse to conclusions of other authors as, for example, Goss and Briddon [2], who argue for a higher stability of B-D complexes. Thus, both the location of hydrogen and the $\mathrm{B}-\mathrm{H}$ complexes binding energy are still under discussion. The presence of hydrogen in some cases even changes the doping character, from p- to n-type, of boron-doped diamond as reported by Teukam et al. [3]. This illustrates the importance of controlling

\footnotetext{
* Corresponding author. Tel.: +34 956 016332; fax: +34956 016471. E-mail address: concha.fernandez@uca.es (C. Fernández-Lorenzo).
}

hydrogen incorporation during and after CVD growth. Recently, Muret et al. [4] reported a highly resistive behaviour located in the last micron of boron-doped CVD grown layers that contrasts with the behaviour of the buried material. Indeed, after plasma etching of $1 \mu \mathrm{m}$ diamond, their epilayer becomes conductive. They attribute this character to a boron passivation by hydrogen or a compensation by donor defects located just in the surface of the epilayers.

In this contribution, we study further this behaviour by means of spectroscopic techniques in order to understand the reasons for such a superficial effect. FTIR (Fourier Transform infrared spectroscopy in reflection mode) allowed the detection of the activated $N_{\mathrm{A}}-N_{\mathrm{D}}$ density, while cathodoluminescence $(\mathrm{CL})$ informs on the activated acceptor density $N_{\mathrm{A}}$ through the relative intensity of free and bound exciton recombinations.

\section{Experimental}

The sample here studied is a boron-doped CVD homoepitaxial diamond. Previous results of conductivity and photoinduced current transient spectroscopy (PICTS) of the presently studied sample have been reported by Muret et al. [4]. The boron-doped film has been deposited on an E6 CVD (100) substrate (optical grade quality, $3 \mathrm{~mm} \times 3 \mathrm{~mm} \times 0.5 \mathrm{~mm}$ ), at a pressure of 50 Torr and a temperature of $910{ }^{\circ} \mathrm{C}$ in a NIRIM type reactor with a $[\mathrm{B}] /[\mathrm{C}]$ ratio of $50 \mathrm{ppm}$, and a $\left[\mathrm{CH}_{4}\right] /\left[\mathrm{H}_{2}\right]$ ratio of $1 \%$. The homoepitaxial layer obtained is $700 \mathrm{~nm}$ thick, with an increasing B-content from $1.5 \times 10^{18} \mathrm{~cm}^{-3}$ at the substrate-epilayer interface to $2 \times 10^{18} \mathrm{~cm}^{-3}$ at the surface. The substrate boron concentration is below the SIMS detection limit $\left(5 \times 10^{15} \mathrm{~cm}^{-3}\right)$. 
To study the buried part of the epilayer, a $500 \mathrm{~nm}$ deep ECR (microwave electron cyclotron resonance plasma of oxygen) etching has been performed on half of the sample surface. A detailed description of the ECR equipment has been already given elsewhere $[5,6]$. The layer was etched at room temperature for 8 min under a $R F$ self-bias voltage of $27.5 \mathrm{~V}$, a microwave power of $1600 \mathrm{~W}$, and an oxygen pressure of 2.35 mTorr.

Room temperature FTIR spectra were collected with a FTIR Nicolet Impact 410 equipment attached to a FTIR microscope IR Plan Advantage using both transmission and reflection modes with a resolution of $4 \mathrm{~cm}^{-1}$. All the spectra were converted into absorbance spectra for calculations. The absolute active boron concentration values were deduced from the absorbance spectra using the calibration of Collins and Williams [7] from the absorption at the $2800 \mathrm{~cm}^{-1}$ absorption line. An identical CVD substrate was used as a reference, to remove the intrinsic diamond related peaks and thus to illustrate the boron-related behaviour.

The CL analyses have been performed at $100 \mathrm{~K}$ and a $5 \mathrm{~K}$ with a Quanta 200 SEM coupled with a collecting mirror and an ANDOR spectrometer system (EM-CCD $1600 \times 400$ pixels and a $160 \mathrm{~mm}$ focal length spectrometer). At $235 \mathrm{~nm}$, the spectral resolution is better than $2 \times 10^{-3} \mathrm{eV}$ for the chosen experimental conditions. The CL spectra were carried out at an acceleration voltage of $20 \mathrm{kV}$ and $5 \mathrm{kV}$ to show effects occurring near the sample surface. All the spectra presented here have been normalized in counts to the $F E_{\mathrm{TO}}$ maxima (free exciton with transverse optical phonon replica) peak.

\section{Results and discussion}

In the following the as-grown region is labelled "region A" and the etched one "region B" as shown in the inset of Fig. 1. First, FTIR absorption spectra were recorded to obtain the absolute active boron concentration. Second, reflectance spectra allowed a much higher spatial resolution but gave only a relative variation of the boronrelated peak. Then, absolute mapping of active boron concentration became possible. Finally, cathodoluminescence measurements were carried out to confirm the FTIR conclusions.

Fig. 1 spectra present absorbance measurements on both regions. Intrinsic diamond shows usually absorption bands in the two and three-phonon range, between 1500 and $4000 \mathrm{~cm}^{-1}$. Those peaks overlap the present boron-related signals attributed to hole transition between dopant levels of the hydrogen-like system. For this reason the intrinsic absorption was subtracted from each spectrum using an identical CVD substrate as reference. The two absorption bands at 2450 and $2800 \mathrm{~cm}^{-1}$, respectively attributed to the ground state to first excited state electronic transition and to the ground state to second excited state electronic transition of neutral boron acceptor [8], are detected only in the region B. These two bands are not detected in region A. Quantifying the total neutral boron present in the substrate + epilayer (region B) system crossed by the optical beam during the transmittance experiment using the integral of the $2800 \mathrm{~cm}^{-1}$ absorption peak, and reducing it to the $200 \mathrm{~nm}$ thick epilayer, a concentration of neutral boron acceptors of around $1 \times 10^{18} \mathrm{~cm}^{-3}$ is deduced. This value is close to that obtained in SIMS experiments: $2 \times 10^{18} \mathrm{~cm}^{-3}$. This is reasonable taking into account that the epilayer thickness of region $B$ after plasma etching is not precisely known. In region $\mathrm{A}$, in spite of an identical boron concentration, there is a total disappearance of both the 2450 and $2800 \mathrm{~cm}^{-1}$ absorption bands (see Fig. 1).

Such results seem to corroborate the Muret et al. [4] results. They found that region A was not conductive while region B behaved as a doped layer. However, they concluded that the surface was probably passivated by hydrogen or compensated by a donor-type defect. The FTIR measurements are performed across the whole sample thickness, thus the conductive/non-conductive transition should not occur depending on the depth in the epilayer. In region A the whole layer

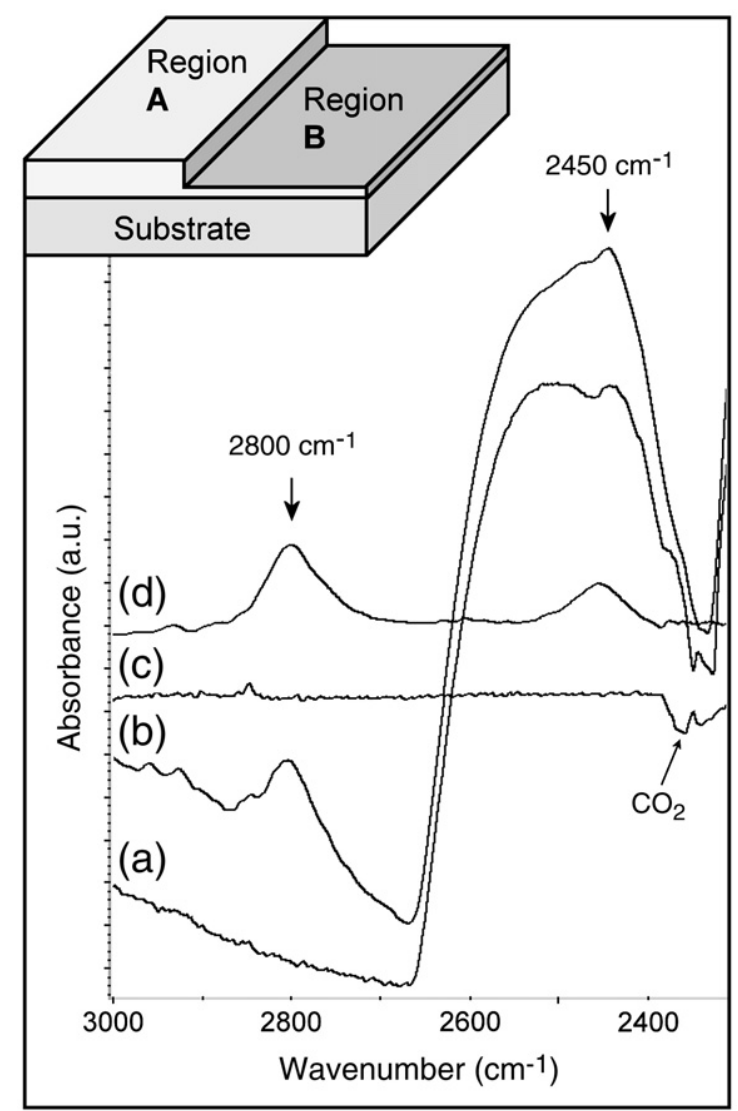

Fig. 1. Absorbance FTIR spectra acquired in (a) as-grown region A and (b) etched region B. The spectra (c) and (d) correspond to the same spectra after subtraction of the substrate contribution. Peaks at $2800 \mathrm{~cm}^{-1}$ and $2450 \mathrm{~cm}^{-1}$ correspond to boron-related hole transitions. Spectrum (c), corresponding to region A, did not reveal the presence of boron while the (d), corresponding to region B, shows both boron-related transitions.

is not conductive as no active boron is present, while in the region $\mathrm{B}$ a large fraction of the boron atoms seem to be electrically active. This seems to indicate that the insulating character of region $A$ is probably due to a hydrogen passivation, as plasma etching cannot remove structural defects without removing the crystal itself.

To investigate this point in more details, FTIR mappings in reflectance mode were carried out. In Fig. 2a an optical microscopy (OM) observation is shown, where the square indicates the reflectance FTIR mapping zone of Fig. 2b. The spectra linescan position of Fig. $2 \mathrm{c}$ is also indicated. The FTIR mapping and spectra are recorded in the reflecting mode giving then relative boron variation information. To obtain the absolute values of active boron incorporation, transmittance measurements on both regions A and B of Fig. 1 were used to "calibrate" the reflectance spectra. In Fig. 2b, the map is obtained using the ratio between the corrected heights at $2800 \mathrm{~cm}^{-1}$ and at $1980 \mathrm{~cm}^{-1}$ (corresponding to the intrinsic two-phonon absorption $\mathrm{LO}+\mathrm{TA}(\mathrm{X})$ ). Both mapping and linescan show the sharp transition between active and non-active boron region. The only difference is the plasma etching operation.

As the absorption coefficient of the $2800 \mathrm{~cm}^{-1}$ absorption peak gives the $N_{\mathrm{A}}-N_{\mathrm{D}}$ concentration [9], this change in the intensity can be due to hydrogen passivation or dopant compensation across the whole thickness. To determine which a mechanism occurs, cathodoluminescence $(\mathrm{CL})$ experiments were carried out. Indeed, the ratio between intensity peaks attributed to free exciton (FE) and bound exciton (BE) recombinations, with the transverse optic (TO) replica, depends directly on the active dopant concentration and can be used to quantify independently donor and acceptor concentrations. Donors induce a $B E_{\mathrm{TO}}$ recombination at $5.175 \mathrm{eV} \mathrm{[10]} \mathrm{while} \mathrm{boron} \mathrm{acceptors}$ 


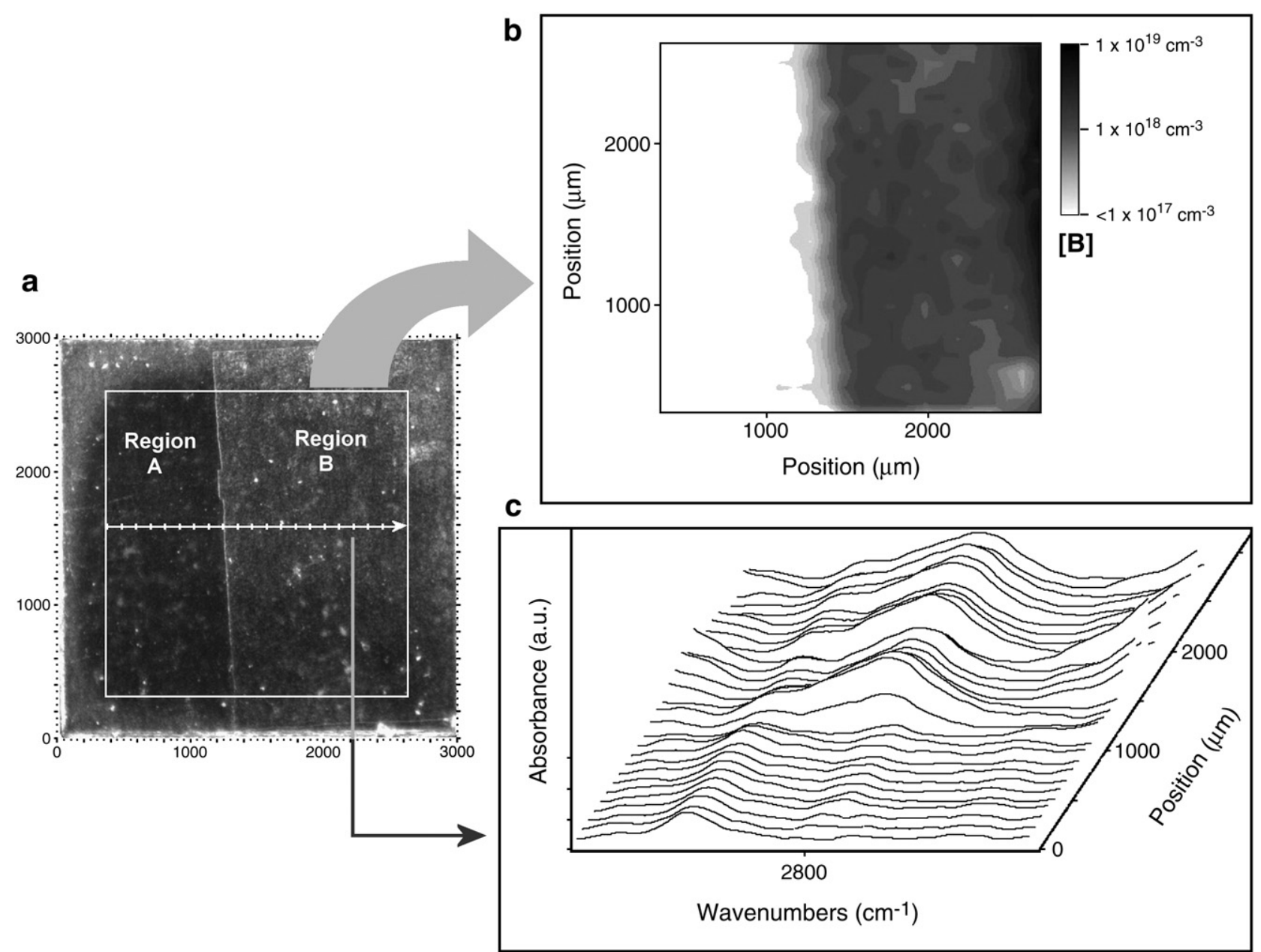

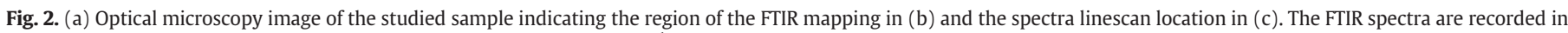

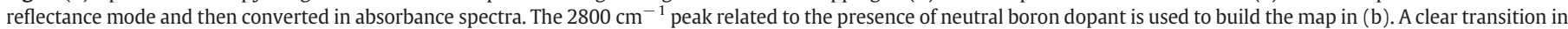
the boron activity is revealed between regions $\mathrm{A}$ and $\mathrm{B}$.

give a $B E_{\mathrm{TO}}^{B}$ recombination at $5.209 \mathrm{eV}$ [11] and both peaks can be observed on the same spectrum [10]. Thus, in the case of a compensation of boron by donors, we expect to observe the two peaks but, if a full passivation of boron occurs, the boron-related $B E_{\mathrm{TO}}^{B}$ excitonic peak is expected to vanish as the excitons cannot anymore be bound to acceptors. Fig. 3 shows the CL spectrum at $5 \mathrm{~K}$ of the $0.2 \mu \mathrm{m}$ thick etched epilayer (region B). The spectrum has been taken at $5 \mathrm{keV}$ to ensure an e-h pair generation mostly in the epilayer. Due to the contribution of the $B E_{T A}^{B}$ component in the recombination at $5.27 \mathrm{eV}$, the $\mathrm{I}\left(B E_{\mathrm{TO}}^{B}\right) / \mathrm{I}\left(F E_{\mathrm{TO}}\right)$ ratio is higher than 12.5 . The $B E_{\mathrm{TO}}^{B}$ peak lays at $5.220 \mathrm{eV}$. The ratio corresponds to the boron concentration saturation value above $7 \times 10^{17} \mathrm{~cm}^{-3}$. The $B E_{\mathrm{T} O}$ peak stands just before the decreasing of the peak energy value occurring above $2 \times 10^{18} \mathrm{~cm}^{-3}$ [12-14]. These results are consistent with that obtained from the FTIR and the SIMS experiments.

Fig. 4a shows the comparison between the two CL spectra at $100 \mathrm{~K}$ obtained on regions A and B for incident electrons of $20 \mathrm{keV}$. A clear absence of the boron acceptor related $B E_{T}^{B}$ peak is shown in region $A$, while this peak is present in region $B$, as already seen in Fig. 3, confirming that active boron is present there. This undoubtedly indicates that boron is passivated in region $\mathrm{A}$ and that no significant compensation occurs. The ratio $\mathrm{I}\left(B E_{\mathrm{TO}}^{B}\right) / \mathrm{I}\left(F E_{\mathrm{TO}}\right)$ in region $\mathrm{B}$ gives a boron concentration of about $1 \times 10^{17} \mathrm{~cm}^{-3}$ lower than that deduced in Fig. 1. This is a consequence of the high electron beam energy used $(20 \mathrm{keV})$ generating e-h pairs in both the substrate and the epilayer. Even though the penetration depth, $R$, reaches $3 \mu \mathrm{m}$, it is important to note that the e-h pair generation is maximum at about the first third of $R[15,16]$. Some of the pairs are generated and recombine in the substrate providing an extra contribution to the $F E_{\mathrm{TO}}$ peak and consequently an underestimation of the $\mathrm{I}\left(B E_{\mathrm{TO}}^{B}\right) / \mathrm{I}\left(F E_{\mathrm{TO}}\right)$ ratio. This

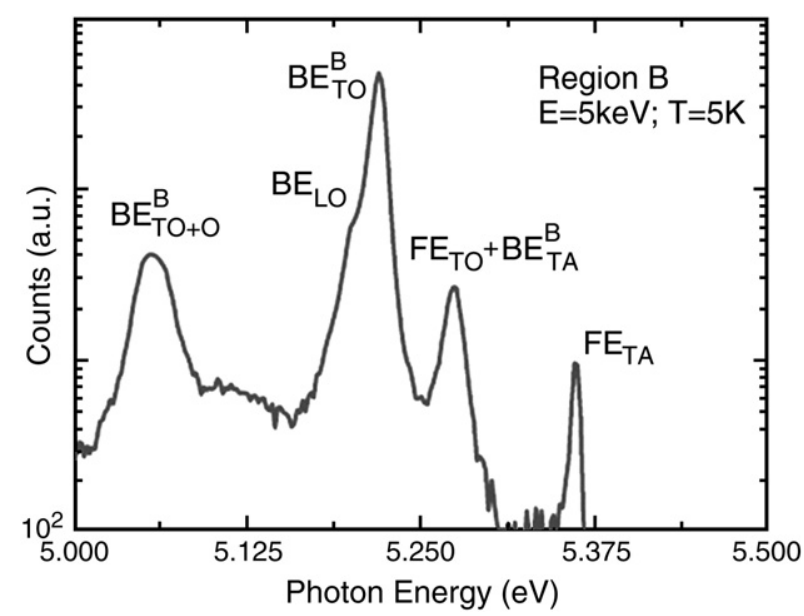

Fig. 3. CL spectrum recorded at $T=5 \mathrm{~K}$ and a beam energy of $5 \mathrm{keV}$ on the plasma etched region B. Assuming a $E_{\mathrm{g}}$ of $5.49 \mathrm{eV}$, an exciton binding of $0.085 \mathrm{eV}$, an acceptor binding of $0.055 \mathrm{eV}$ and a phonon energy of $\hbar \omega_{\mathrm{TA}}=0.087 \mathrm{eV}$ and $\hbar \omega_{\mathrm{TO}}=0.141 \mathrm{eV}$ for acoustic and optical transverse phonons, the $B E_{\mathrm{TA}}$ and $F E_{\mathrm{TO}}$ transitions stand at $5.263 \mathrm{eV}$ and $5.268 \mathrm{eV}$ respectively. 


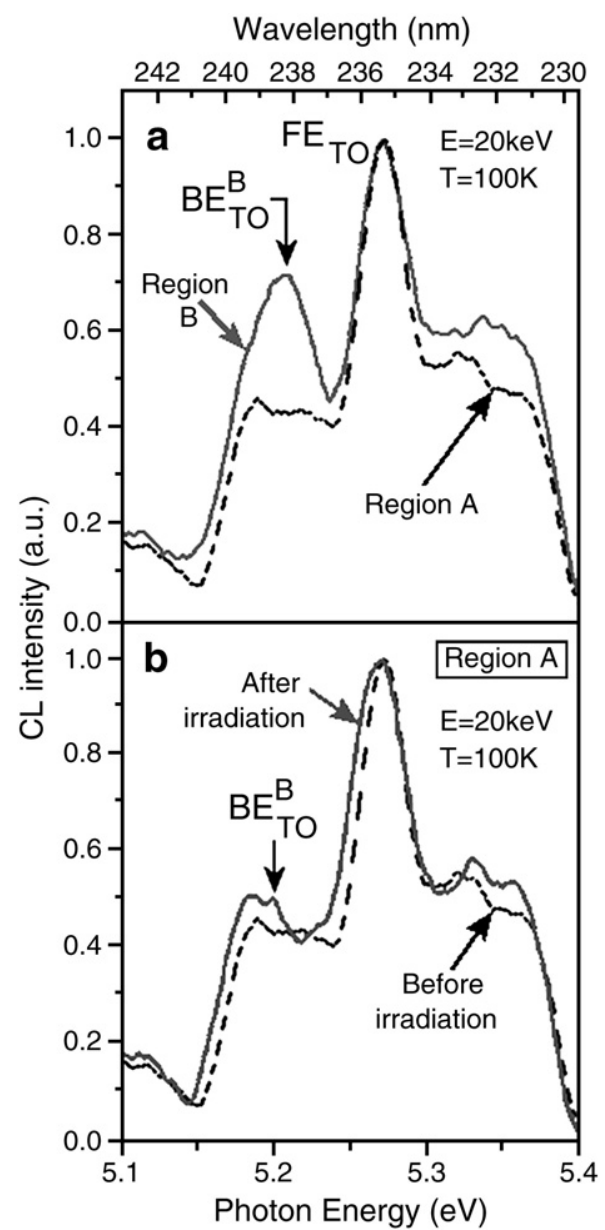

Fig. 4. CL spectra recorded at a temperature of $100 \mathrm{~K}$ and a beam energy of $20 \mathrm{keV}$ and normalized in counts to the $F E_{\mathrm{TO}}$ maxima peak intensity. (a) Spectra recorded on regions $A$ and $B$ showing a clear difference in the $B E_{\mathrm{TO}}^{B}$ intensity. The relative importance of the $B E_{\mathrm{TO}}^{B}$ peak in region $B$ is shown to decrease with respect to that of Fig. 3 recorded at $5 \mathrm{keV}$. This mainly results from the higher beam energy that induces a smaller proportion of e-h generation into the boron-doped epilayer. The absence of $B E_{\mathrm{T} O}^{B}$ in region $\mathrm{A}$ is due to a hydrogen passivation of boron acceptors. (b) Spectra recorded before and after electron irradiation of region A. A slight reactivation of passivated boron is observed (see arrow).

explains why the boron concentration deduced from CL with $20 \mathrm{keV}$ incident electrons in region $\mathrm{B}$ is significantly underestimated.

On the other hand, boron acceptors can be reactivated by electron irradiation as recently reported [1]. When irradiating with electrons the passivated region A, using the experimental conditions $(T=100 \mathrm{~K}$, $t=15 \mathrm{~min}$., beam conditions: $10 \mathrm{keV}, 57 \mathrm{nA}$, on square surface of $25 \mu \mathrm{m}^{2}$ ) reported by Barjon et al. [1], some reactivation is observed (see arrow in Fig. 4b). The relatively reduced $B E_{\mathrm{TO}}$ peak intensity after irradiation suggests a lower reactivation than that reported by Barjon et al. We tentatively attribute it to a different hydrogen incorporation mechanism than that occurring during diffusion. Indeed, in this case, the incorporation takes place during CVD growth. The hydrogen location and, thus, its binding energy might be higher, making the $\mathrm{B}-\mathrm{H}$ complex more stable. The exact mechanism of B-H complex dissociation of the underlying diamond epilayer during ECR $\mathrm{O}_{2}$ plasma etching remains to be determined.

\section{Conclusions}

FTIR and CL experiments were carried out on a boron-doped homoepitaxial layer. Previously published results point out to hydrogen passivation or defect related compensation taking place near the surface. The present investigation highlights the mechanisms here involved: hydrogen passivation during growth is responsible for the insulating character of the $700 \mathrm{~nm}$ thick epilayer doped with $2 \times 10^{18} \mathrm{~cm}^{-3}$ boron. The plasma etching applied on part of the sample surface seems to induce a dissociation of the B-H complexes which would explain the conductive character of the plasma etched region. The B-H complexes seem to be more stable in this growth-related mechanism than upon the usual plasma hydrogen diffusion. Finally, the FTIR mapping method is revealed to be a very powerful technique to determine absolute densities of active dopants in diamond.

\section{Acknowledgments}

We thank the Ministerio de Ciencia e Innovación (MICINN) of the Spanish Government for funding under Grant No. TEC2009-11399. We are also grateful for the use of the Central Electron Microscopy Facilities of the University of Cádiz.

\section{References}

[1] J. Barjon, A. Mehdaoui, F. Jomard, J. Chevallier, C. Mer, M. Nesladek, P. Bergonzo, J. Pernot, F. Omnès, A. Deneuville, Appl. Phys. Lett. 93 (2008) 62108

[2] J.P. Goss, P.R. Briddon, Phys. Rev. B 77 (2008) 35211.

[3] Z. Teukam, J. Chevallier, C. Saguy, R. Kalish, D. Ballutaud, M. Barbé, F. Jomard, A. Tromson-Carli, C. Cytermann, J.E. Butler, M. Bernard, C. Baron, A. Deneuville, Nat. Mater. 2 (2003) 482.

[4] P. Muret, A. Kumar, P.N. Volpe, M. Wade, J. Pernot, L. Magaud, C. Mer, P. Bergonzo, Phys. Status Solidi A 1-6 (2009) 2016.

[5] M. Bernard, A. Deneuville, T. Lagarde, E. Treboux, J. Pelletier, P. Muret, N. Casanova, E. Gheeraert, Diamond Relat. Mater. 11 (2002) 828.

[6] M. Bernard, A. Deneuville, L. Ortega, K. Ayadi, P. Muret, Diamond Relat. Mater. 13 (2004) 287.

[7] A.T. Collins, A.W.S. Williams, J. Phys. C: Solid State Phys. 4 (1971) 1789.

[8] J. Chevallier, A. Lusson, D. Ballutaud, B. Theys, F. Jomard, A. Deneuville, M. Bernard, E. Gheeraert, E. Bustarret, Diamond Relat. Mater. 10 (2001) 399.

[9] K. Thonke, Semicond. Sci. Technol. 18 (2003) S20.

[10] D. Araujo, A. Tajani, E. Gheeraert, E. Bustarret, J. Phys.: Condens. Matter 16 (2004) S287.

[11] D. Araujo, M. Kadri, M. Wade, E. Bustarret, A. Deneuville, Phys. Status Solidi C 2 (4) (2005) 1336.

[12] C. Baron, A. Deneuville, M. Wade, F. Jomard, J. Chevallier, Phys. Status Solidi A 203 (3) (2006) 544.

[13] M. Kasu, M. Kubovic, A. Aleksov, N. Teofilov, Y. Taniyasu, R. Sauer, E. Kohn, T. Makimoto, N. Kobayashi, Diamond Relat. Mater. 13 (2004) 226.

[14] H. Kawarada, A. Yamaguchi, Diamond Relat. Mater. 2 (1993) 100.

[15] D. Araújo, J.-M. Bonard, G. Oelgart, J.-D. Ganière, F. Morier-Genoud, F.K. Reinhart, Mater. Sci. Eng., B 28 (1993) 124.

[16] J.-M. Bonard, J.-D. Ganière, B. Akamatsu, D. Araújo, F.K. Reinhart, J. Appl. Phys. 79 (1996) 8693. 